\title{
CIRUGÍA ESTÉTICA Y MOTIVACIONES PSICOSOCIALES. HACIA UN ESTADO DE LA CUESTIÓN Y PERSPECTIVAS DE INVESTIGACIÓN
}

\author{
Luis Pérez García ${ }^{1}$ \\ https://orcid.org/0000-0001-7553-5067 \\ Alicia Almanzar Curiel ${ }^{2}$ \\ https://orcid.org/0000-0002-5993-5818
}

\begin{abstract}
RESUMEN
Se observa que en la modernidad tardía la cirugía estética está experimentando un incremento constante cada año. A pesar de ser un área de estudio relativamente joven, ya existe una vasta y rica literatura que aborda la decisión de someterse a cirugía estética desde la perpectiva psicosocial. En relación con la cirugía estética, las motivaciones psicosociales más estudiadas son la imagen corporal, el trastorno dismórfico corporal, la identidad, el manejo del terror, la religión, etc. Para ello se consultaron autores como: Sarwer, et al. (1998), Behravan (2018), International Society of Aesthetic Plastic Surgery $(2015,2018,2019)$, entre otros. En México existen muy pocas investigaciones sobre las motivaciones psicosociales de cirugía estética. El presente trabajo se propone como objetivo una revisión del estado del conocimiento sobre algunas de las motivaciones arriba mencionadas, con la intención de abrir el camino en la generación de conocimiento en este campo de estudio. La búsqueda de los materiales adecuados en el Internet se realizó a través de Google Académico excluyendo todas aquellas que no son de acceso abierto. Del análisis de los materiales encontrados se recuperan algunas de las aportaciones importantes, y se desprenden preguntas e hipótesis para investigaciones futuras. Finalmente, es importante decir que las motivaciones psicosociales de cirugía estética, por razones analíticas, se observan por separado, aunque en la realidad concreta suelen encontrarse interconectadas entre sí, lo que también amplia el panorama de investigación.
\end{abstract}

Palabras clave: México; modernidad tardía; cirugía estética; motivaciones psicosociales.

\section{Cirurgia estética e motivações psicossociais. Estado da questão e perspectivas de investigação.}

\section{RESUMO}

Observa-se que na modernidade tardia, a cirurgia estética está experimentando um incremento constante em cada ano. Apesar de ser uma área de estudo relativamente nova, já existe uma vasta e rica literatura que aborda a decisão de submeter-se a cirurgia estética sob uma perspectiva psicossocial. Em relação com a cirurgia estética, as motivações psicossociais mais estudadas são: a imagem corporal, o transtorno dismórfico corporal, a identidade, o manejo do terror, a religião, entre outras. Para o efeito consultaram-se autores como: Sarwer, et al. (1998), Behravan (2018), International Society of Aesthetic Plastic Surgery (2015, 2018, 2019), entre outros. No México existem poucas investigações sobre as motivações psicossociais de cirurgia estética. O presente trabalho propõe-se realizar uma revisão do estado de conhecimento sobre algumas das motivações supra mencionadas, com a intenção de abrir o caminho na geração de conhecimento nesse campo de estudo. A busca dos materiais adequados na Internet realizou-se através de Google Académico excluindo todas aquelas que não são de Acesso Aberto. A partir da análise dos materiais encontrados recuperam-se algumas contribuições importantes e surgem questões e hipóteses para investigações futuras. Finalmente, é pertinente dizer que as motivações psicossociais de cirurgia estética, por razões analíticas, observam-se de forma separada, apesar de que na realidade concreta, geralmente se encontram interconectadas entre sí, o que também amplia o panorama de investigação.

Palavras-chave: México; modernidade tardia; cirurgia estética; motivações psicossociais.

Cosmetic surgery and psychosocial motivations. Towards a state of the art and research perspectives.

\section{ABSTRACT}

It is observed that in late modernity cosmetic surgery is experiencing a constant increase every year. Despite being a relatively young area of study, there is already a vast and rich literature that addresses the decision to undergo cosmetic surgery from a psychosocial perspective. In relation to cosmetic surgery, the most studied psychosocial motivations

${ }^{1}$ Universidad de Guadalajara, México. E-mail: luisperezg@hotmail.com

${ }^{2}$ Universidad de Guadalajara, México. E-mail: aliciaalmanzarcuriel@yahoo.com.mx 
are body image, body dysmorphic disorder, identity, handling of terror, religion, etc. For this, were consulted authors such as: Sarwer, et al. (1998), Behravan (2018), International Society of Aesthetic Plastic Surgery (2015, 2018, 2019), among others. In Mexico there is very little research on the psychosocial motivations for cosmetic surgery. The present work proposes as objective a review of the state of knowledge about some of the above-mentioned motivations, with the intention of opening the way in the generation of knowledge in this field of study. The search for suitable materials on the internet was carried out through academic google, excluding all those that are not open access. From the analysis of the materials found, some of the important contributions are recovered, and questions and hypotheses emerge for future research. Finally, it is important to say that the psychosocial motivations for cosmetic surgery, for analytical reasons, are observed separately, although in concrete reality they are usually interconnected, which also broadens the research panorama.

Keywords: México; late modernity; plastic surgery; psychosocial motivations.

\section{Introducción}

La diferencia fundamental entre la cirugía plástica-reconstructiva y los procedimientos cosmetológicos (quirúrgicos y no quirúrgicos) radica en que la primera se realiza por razones médicas y las segundas para mejorar la belleza corporal en general (cuerpo, cabeza y cara). Las cirugías reconstructivas son el antecedente inmediato de las cirugías estéticas. En un principio, la cirugía plástica se realizaba para reparar partes del cuerpo que habían sido alteradas o afectadas en su funcionamiento, por ejemplo, por la ira del marido, las guerras, los duelos, las peleas callejeras, etc.

En relación con la cosmetología quirúrgica, inicialmente los principales consumidores fueron cantantes, actores, actrices, etc. y gente que podía pagar. Se buscaba fundamentalmente ocultar el envejecimiento del cuerpo. Ahora es una práctica más popular. Desde el inicio de la modernidad tardía en 1960 con los cambios sociales y tecnológicos, y hasta nuestros días, la cirugía estética se está volviendo cada vez más popular en hombres y mujeres de todas las edades. Aunque los hombres poco a poco se están sumando a las estadísticas, siguen siendo las mujeres quienes más solicitan este tipo de servicios (Davis, 2002). Cabe decir que, en tiempos recientes, para alcanzar la belleza corporal también se están empleando otros procedimientos cosmetológicos no quirúrgicos o de mínima invasión, por ejemplo, el Botox.

La International Society of Aesthetic Plastic Surgery (ISAPS) es la única organización que genera datos estadísticos de los procedimientos cosméticos, quirúrgicos y no quirúrgicos, que se realizan a nivel mundial. Para recolectar la información envía cuestionarios a 35 mil cirujanos plásticos de todo el mundo que forman parte de una base de datos propiedad de ISAPS.

Comparando los datos de las encuestas de 2015 y 2019 se observa un continuo incremento de los procedimientos cosmetológicos en el mundo. Estados Unidos sigue siendo uno de los principales consumidores, seguido de Brasil, Japón, México, etc. Comparando las intervenciones quirúrgicas de 2015 y 2019 (9,641,253 y 11,363,569 intervenciones respectivamente), el incremento fue 17.86 por ciento. Con respecto a los procedimientos no quirúrgicos en los mismos años $(12,055,418$ y 13,618,735 intervenciones respectivamente), el incremento fue de 12.96 por ciento (ISAPS, 2015, 2019).

Por país, los datos de 2018 y 2019 muestran que México ocupa el tercer y cuarto lugar respectivamente a nivel mundial. En total en 2018 se realizaron 1,043,247 procedimientos cosmetológicos (equivalente al 4.5 por ciento del total mundial), de los cuales 518,046 fueron quirúrgicos, y 525,200 no quirúrgicos. En 2019 el total de procedimientos cosmetológicos se incrementó en 15.07 por ciento (ISAPS 2018, 2019).

Los procedimientos más solicitados van a depender del sexo y la edad del paciente. A pesar de que los hombres poco a poco están tomando la decisión de someterse a procedimientos cosmetológicos, las mujeres siguen siendo las principales consumidoras. Lo que más solicitan las mujeres es el aumento de pecho, aunque disminuyó 3.6 por ciento en comparación con 2018. En general, los cinco procedimientos quirúrgicos más solicitados son el aumento de pecho (mamoplastia de aumento), la liposucción, la cirugía de párpados, la abdominoplastia y la 
rinoplastia; y en los no quirúrgicos se destaca la toxina botulínica, el ácido hialurónico, la eliminación de vello, la reducción de grasas y la foto rejuvenecimiento.

En su conjunto, de los procedimientos quirúrgicos, el de nalgas también es uno de los más solicitados (creció en un 38.4 por ciento), junto con la labioplastia que se incrementó en 24.1 por ciento. Por género, los tres procedimientos quirúrgicos más solicitados por las mujeres siguen siendo el aumento de pecho, la liposucción y la cirugía de párpado, y en los hombres la ginecomastia, la cirugía de párpado y la liposucción. En ambos géneros se practica la toxina botulínica, el ácido hialurónico y la eliminación de vello.

Por edades, el consumo de cirugías estéticas se da más entre mujeres de 35 a 50 años, aunque el aumento de pecho y las rinoplastias se concentran en las edades de 19 a 34 años. Los procedimientos inyectables con toxinas botulínicas se practicaron más en las edades de 35 a 50 años, y entre los pacientes de 18 años o menores las rinoplastias siguen siendo las más comunes. En México, las tres cirugías estéticas más solicitadas por sección corporal, son las siguientes. En cara y cabeza: lifting de cejas, cirugía de orejas, y cirugía de párpados. En senos: aumento de senos, extracción de implantes mamarios, y levantamiento de senos. En cuerpo y extremidades: abdominoplastia, aumento de glúteos, y levantamiento de glúteos. Con respecto a los procedimientos no quirúrgicos, los tres más populares según su tipo son los siguientes. Inyectables: toxina botulínica, hidroxiapatita de calcio, y ácido hialurónico. Rejuvenecimiento facial: peeling químico, ablativo de campo completo, y rejuvenecimiento micro ablativo (ISAPS, 2019).

Los datos estadísticos presentados no solo muestran que son cada vez más las personas que desean parecer más jóvenes modificando alguna parte de la superficie de su cuerpo, sea de manera quirúrgica o no quirúrgica. También hacen suponer que las personas toman estas decisiones porque no están contentas con alguna parte de su cuerpo. En la siguiente sección se plantea el contexto social que ayuda a explicar el bum de la cirugía estética en el mundo, y también se plantea la pregunta de investigación.

\section{Contexto y pregunta de investigación}

Para explicar el auge de los procedimientos cosmetológicos, Askegaard, et al. (2002), consideran que, a diferencia de otras épocas, en la modernidad tardía son muy pocas las personas que rechazarían someterse a una cirugía estética con la finalidad de mejorar tanto las apariencias innatas o dejadas por las vidas vividas como la salud psicológica; también señalan que los procedimientos quirúrgicos, ya no son solo para unos cuantos, millones de personas en el mundo, de diferentes edades, y de todos los géneros con capacidad de pago, están haciendo fila esperando una intervención en busca del cuerpo perfecto; y finalmente, subrayan que en esta época se está volviendo cada vez más aceptado tratar de corregir una imagen corporal y personal problemática mediante la cirugía estética.

Otros factores que inciden en el incremento de procedimientos cosmetológicos son los grandes avances en las técnicas y tecnologías para "corregir" los desperfectos del cuerpo; su publicidad en los mass media; y la competencia natural por la belleza corporal. Los avances técnicos y tecnológicos permiten que muchas de las intervenciones se realicen solamente con anestesia local, lo que reduce el costo y el temor en los pacientes. Los procedimientos endoscópicos no invasivos también permiten una pronta recuperación y resultados con apariencia natural, olvidando las grandes heridas y largos procesos de sanación.

La publicidad en los medios, por su parte, enseña a negar nuestra corporalidad - por ejemplo, las narices grandes, los vientres voluminosos, los senos pequeños, las pompis pequeñas y flácidas, las piernas mal torneadas, las arrugas de la cara, los parpados caídos, la papada grande, etc.-, y nos invita a cambiarla argumentando que el moldeo de los cuerpos imperfectos no solo los vuelve más atractivos, también mejora la autoestima, atrae las miradas de aprobación, logra obsequios caros, y amplia la lista de quienes verifican la belleza corporal (Blum, 2003: 7). 
Con respecto a la competencia por la apariencia más bella, esta ocurre actualmente en tres espacios: en la vida real, en la realidad virtual y en los medios de comunicación (Behravan, 2018: 10). Para atraer las miradas, el ser humano ha ido construyendo a lo largo de la historia andamiajes estéticos, tanto técnicos como culturales centrados en lo visual, aunque en la modernidad tardía el placer que produce, por ejemplo, la visualización de la belleza lograda mediante cirugía, es aún más importante, sobre todo en las mujeres (De Castro, 2009).

Hasta aquí, se ha mostrado el contexto social en el cual se está dando el boom de los procedimientos cosmetológicos en el mundo. En torno al fenómeno, desde diferentes perspectivas teóricas y metodológicas, entre otras cosas, se ha investigado qué motiva a los individuos (hombres y mujeres) a someterse a una u otra cirugía estética; los pensamientos y sentimientos antes, durante y después de las intervenciones; la influencia en la vida e identidad personal; la mejora de la salud, la calidad de vida, la autoestima, y la auto-imagen corporal después de la cirugía; etc.

Otros estudios han centrado su atención en las dimensiones psicosociales de la cirugía estética, a saber, imagen corporal, trastorno dismórfico corporal, identidad, manejo del terror, religión, perfeccionismo, sentido de pertenencia, bienestar subjetivo, autoestima, conservadurismo, y toma de riesgos (Behravan, 2018). En México, este tipo de estudios son escasos. Con la finalidad de ir cubriendo este vacío, el presente trabajo pretende recuperar algunos de los conocimientos iniciales que sobre las motivaciones psicosociales mencionadas y la cosmetología propiamente quirúrgica se han generado en distintas partes del mundo, principalmente en idioma inglés. Con el análisis de los materiales no solo se podrán observar los esfuerzos iniciales y avances en el desarrollo del conocimiento sobre cada una de las dimensiones psicosociales de la cirugía estética, sino también las áreas en donde los conocimientos son más abundantes que en otros. De la revisión también se espera desprender algunas preguntas e hipótesis para futuras investigaciones.

\section{Metodología}

La búsqueda de los materiales adecuados se realizó a través de la técnica manual en donde las lecturas realizadas van llevando a los autores seminales. La búsqueda de materiales se realizó en la internet, principalmente en google académico, PUBMED y también en internet abierto, utilizando, en español e inglés, las palabras clave "cirugía estética", y la motivación psicosocial que en turno se este indagando, por ejemplo, "imagen corporal". En principio, de la literatura localizada, se incluyeron las publicaciones en revistas arbitradas o indexadas en bases electrónicas de acceso abierto en internet; las que en su título hacen referencia tanto a la cirugía estética como a alguna de las motivaciones psicosociales aquí en cuestión, independientemente de su enfoque teórico y metodológico; y las que teorizan sobre alguna de las motivaciones psicosociales, dando prioridad a aquellas que iniciaron con la discusión el tema, o las más representativas, y a otras posteriores que las complementan o critican. Se excluyen las publicaciones que, aunque pertinentes, no son de acceso abierto. Finalmente, el proceso de selección tomó en cuenta para su análisis 52 referencias.

\section{La cirugía estética y las motivaciones psicosociales}

Las motivaciones psicosociales son un proceso mediador que integra componentes subjetivos (vgr. expectativas, deseos, satisfacción, sentimientos) y objetivos (contexto en el cual se interacciona y actúa). Grosso modo, se puede decir que la motivación psicosocial es un estado interno que experimenta el individuo en relación a algo, pero expuesto a factores externos, por ejemplo, otras personas, instituciones, normas y reglas, creencias, tradiciones, medios de comunicación, redes sociales en la internet, etc., que forman parte del contexto social. Así dicho, unas motivaciones pueden consideradas de carácter más individual, por ejemplo, la autoestima, y otras de carácter más social, por ejemplo, la religión. En las siguientes líneas, en relación con la cirugía estética, de manera breve por el espacio que aquí se tiene, se analizan las siguientes motivaciones psicosociales: imagen corporal, trastorno dismórfico corporal, identidad, manejo del terror, religión, y 
perfeccionismo, tratando de desprender en cada caso algunas preguntas e hipótesis interesante para futuras investigaciones.

\section{Imagen corporal y cirugía estética}

Desde un marco psicológico y sociológico de las percepciones y las experiencias de la imagen corporal, con su obra Imagen y apariencia del cuerpo bumano: estudios sobre energías constructivas de la psique publicada en 1935, Paul Schilder fue el pionero en el desarrollo del concepto. Para este autor, la imagen corporal es la imagen que en sociedad cada individuo va construyendo en su mente de su propio cuerpo, es decir, la forma en que imaginamos nuestro cuerpo o deseamos ser, misma que no corresponde regularmente con nuestra imagen real. Cuando esto último ocurre en un individuo concreto, sostiene Grogan (1999), se está ante la autonegación del cuerpo propio, lo que muy probablemente afecte la autoestima y conlleve a trastornos psicológicos, incluso al suicidio (Behravan, 2018: 64).

Mas recientemente, Cash (2004), en su teorización de la imagen corporal, también considera que el cuerpo es una construcción socio-natural y por ello es importante para comprender el comportamiento humano. Desde esta perspectiva también se vuelve relevante estudiar la imagen corporal en adolecentes porque en los casos donde se tiene una autoimagen negativa pueden afectarse sentimientos, comportamientos, pensamientos e interacciones, lo que afectaría la calidad de vida de este grupo de edad en el futuro, volviéndose candidatos probables de cirugía estética. En relación con la elección de cirugía estética, el sentido que de la propia apariencia física va construyendo un individuo, frente a las imágenes de otras personas, incluidas las que promueven los mass media como "imagen ideal", puede contribuir a que busque cambiar su imagen real por otra ideal a través de la cirugía estética (Sarwer et al., 1998). Los resultados en estos casos pueden mejorar la autoimagen negativa, y con ello también mejorar significativamente la autoestima (Sarwer et al., 2005), aunque no se descartan los casos en que el resultado no es el esperado. Para avanzar en este problema, futuros estudios podrían incorporar al análisis la insatisfacción con la imagen corporal cuando se presuma una autoimagen distorsionada (Behravan, 2018: 68), y también las variables sociodemográficas género, edad, estado civil, escolaridad, empleo, ideología de género, religiosidad, valores personales y clase social.

\section{Trastorno dismórfico corporal y cirugía estética}

El trastorno dismórfico corporal (BDD por sus siglas en inglés) es un trastorno mental caracterizado por una insatisfacción extrema con la imagen corporal propia, categoría diagnóstica presente en el Diagnostic and Statistical Manual of Mental Disorders, DSM-5, (American Psychiatric Association 2013), integrada al espectro obsesivo-compulsivo con diversos grados de introspección junto al 'trastorno dismórfico muscular'.

El BDD es la preocupación que un individuo puede tener por un defecto de su apariencia imaginado, mismo que puede ir de leve a excesivo. Para calificarse como tal, la preocupación debe causar angustia o deterioro significativo en las áreas sociales, laborales u otras áreas de interacción de los individuos en la vida cotidiana. Los defectos pueden "verse" en cualquier área del cuerpo, pero las áreas más comunes son piel, cara, abdomen, senos y nariz.

Con mucha frecuencia los individuos con BDD buscan atención médica, y entre ellas puede estar la cirugía estética (Phillips, 1993; Andreasen y Bardach, 1977; Veale et al., 1996). En general se considera que las mujeres presentan mayor insatisfacción con la apariencia física (Phillips y Diaz, 1997), y que por ello son ellas las que más acuden a cirugía estética. Un vacío de conocimiento en estas investigaciones radica en que no se diferencia por sexo a los pacientes con BDD que eligen cirugía estética. Futuros estudios podrían intentar equilibrar la muestra para hacer comparaciones por género, y saber si realmente las mujeres son las que presentan mayor problema de BDD, y en qué casos los hombres también. 
Otra área de investigación interesante son los resultados posteriores de los pacientes con BDD que deciden someterse a cirugía estética. Los estudios muestran que la mejora de la apariencia realmente no produce ningún cambio en la gravedad de los síntomas del trastorno psicológico (Crerand, et al., 2005; Phillips, et al., 2001), incluso algunas personas desarrollan nuevos problemas de apariencia después del tratamiento (Behravan, 2018: 72). Futuros estudios podrían analizar los resultados posteriores observando si se presentan diferencias al agregar las variables sociodemográficas.

\section{Identidad y cirugía estética}

Con su obra Identity. Youth and Crisis, publicada en 1968, Erick H. Erikson se convierte en pionero en el tema de la identidad en la juventud. Desde una perspectiva freudiana de la relación entre el conflicto neurótico y los conflictos normativos que todo niño debe atravesar, y cuyos residuos lleva el adulto en lo más hondo de su personalidad, la identidad funciona como una parte importante que contribuye a mejorar la personalidad del adolescente, paso vital para llegar a la edad adulta como un individuo feliz y eficiente (Feixa, 2020). Berzonsky $(1988,1990)$, tratando de ir más allá de la crisis normativa de Erikson, considera que la identidad es la percepción que vamos construyendo nosotros mismos de quién somos, resultante de la integración de las experiencias pasadas, presentes y futuras.

Así dicho, la identidad es un ejercicio de autorreflexión a través del cual un individuo pondera sus capacidades y potencialidades, y se reconoce consiente de lo que es. En tanto el individuo no vive solo, el autoconocimiento implica también necesariamente reconocerse como miembro de un grupo, dentro del cual se reconoce como es, de frente y en interacción con otros semejantes, miembros de su grupo de pertenencia, y con otros diferentes, miembros de otros grupos. La identidad por tanto también tiene una dimensión colectiva que se puede observar tanto en relación directa con las narrativas de los sujetos sobre el entorno y sobre sí mismos en las interacciones sociales, que son eminentemente simbólicas, y en los movimientos sociales. Desde esta perspectiva teórica, la identidad de cada individuo se construye en un contexto histórico particular (situado), a lo largo de un proceso constante, donde los sujetos elaboran, reelaboran y dan sentido a los elementos culturales del grupo de pertenencia, definiendo en este mismo proceso también su grado de identificación con el grupo de pertenencia, así como de distancia de miembros de otros grupos (Portal, 1991:3-5).

Es importante subrayar que los individuos son susceptibles de cambiar su identidad, incluso por otra muy diferente y opuesta al grupo original de pertenencia, solo que para ello se requiere que los individuos mismos, en sociedad, la modifiquen, es decir, que lleguen a la conclusión de que sus recetas previas para resolver problemas en la vida diaria han dejado de ser útiles o eficaces, lo que significa también que el " $m \imath$ " ha dejado de verse en el "otro generalizado" (normas y reglas sociales de comportamientos dominantes en un espacio y un tiempo determinado), imponiéndose el "yo" hasta que otro "mí" se consolide (Mead, 1973).

Teniendo en mente esta amplia introducción a la identidad personal, enseguida se trata su relación con la elección de cirugía estética. En el proceso de autoconocimiento de uno mismo y de nuestro entorno también se incluyen nuestros rasgos físicos y los de otras personas, y es así como se va constituyendo también nuestra identidad corporal.

Berzonsky (1988, 1990), retomando a Erikson, ha trabajado con los patrones de identidad que podrían estar atrás tanto de la elección de cirugía estética como de su rechazo. En principio, considera que la identidad es un proceso cognitivo, es decir, interiorizado mediante procesos de experienciación y de socialización reflexivos que van constituyendo y definiendo el yo, y también su pertenecía y su distinción de otros. En su esquema teórico, los individuos, motivados por su identidad pueden disentir o diferenciarse de otros individuos en los procesos sociocognitivos que utilizan para resolver problemas personales, toma de decisiones y procesamiento de información "relevante", por ejemplo, acerca de las cirugías estéticas. Así, Berzonsky desarrolla tres estilos de 
procesamiento de identidad (informativo, normativo y difuso/evitativo) que pueden ser útiles para el estudio de la elección de cirugía estética.

Los individuos con estilo de identidad informativa serían aquellos que investigan, evalúan y utilizan información relevante para ellos; ponen en duda sus propias autoconstrucciones y están dispuestos a probar y revisar aspectos de su propia identidad cuando se enfrentan a comentarios (Berzonsky, 1990). Su identidad en este caso puede clasificarse como lograda o en proceso de lograrla (Marcia, 1966; Berzonsky y Neimeyer, 1994). También se caracterizan por profundizar en el ser de las cosas, afrontar directamente los problemas, tomar decisiones cuidadosas o prudentes, ser agradables y tener regularmente éxito en todo lo que emprenden (Berzonsky, 1990, 1992).

En el estilo de identidad normativa se encuentra los que individuos tienden a actuar ajustándose a las instrucciones y expectativas de quiénes son importantes en sus vidas. También tienen baja tolerancia a la ambigüedad; confían fuertemente en sus ideas, creencias y conocimientos para resolver problemas; e interiorizan fácilmente valores y creencias de otros sin cuestionarlos siempre y cuando consideren que no contradice su propio sistema de creencias y valores (Berzonsky, 1992; Berzonsky y Kuk, 2000).

Finalmente, el etilo de identidad difusa/evasiva es propio de los individuos reacios a encarar y afrontar problemas personales, y si lo hacen, es después de haberse demorado los suficiente, y más por las presiones externas o los incentivos situacionales que les ofrecen. Este tipo de identidad se asocia positivamente con estrategias de decisión de afrontamiento evitativo, deficiencia personal, y desadaptativo, y negativamente con autorreflexión y persistencia cognitiva (Berzonsky, 1994, 1998; Berzonsky y Ferrari, 2009).

De acuerdo con Behravan (2018), los patrones de identidad tienen su efecto a través de la salud mental. En otras palabras, en la etiología de la enfermedad mental están presentes los problemas de identidad con uno mismo. En la psiquiatría y en la psicología clínica se acepta que la salud mental del individuo se asocia, al menos en parte, con autoconcepciones positivas, alta autoestima y/o posesión de una identidad que los demás valoran (Behravan, 2018: 79). En cambio, los trastornos psicológicos se asocian a conflictos inconscientes con la personalidad (Freud, 19141918); al desarrollo inadecuado o detenido de la identidad, como es el caso de la identidad normativa de acuerdo con los estudios de Erikson; a los entornos que amenazan la autoconcepción o la autoestima, y al cambio de identidad hacia otro más inestable (Thoits, 1995). Y en general, la baja autoestima, los sentimientos de inutilidad, y la imagen inestable de uno mismo, son factores fundamentales para identificar depresión mayor, trastorno bipolar, distimia (trastorno afectivo o del estado de ánimo) y trastornos límite y de personalidad (Behravan, 2018: 79).

Utilizando el esquema teórico anterior se puede suponer que existe correlación entre estilos de identidad y salud mental, particularmente, que la depresión, la baja autoconfianza, la ansiedad y algunos problemas de conducta correlacionan con el estilo de identidad evasivo/difuso. Al respecto Berzonsky (2003) mostró que la falta de compromiso serio colocaría a quiénes tienen estilos de identidad evasivos/difusos en una situación muy vulnerable.

Actualmente, no existen investigaciones que arrojen luz sobre los tipos de identidad, la salud mental y la elección, o no, de cirugía estética (Behravan, 2018: 82). En lo general, los pacientes que solicitan cirugía estética son individuos ansiosos (Meningaud, et al., 2001), lo que se manifiesta en falta de confianza en sí mismos frente a la sociedad, y miedo de ser evaluados como feos por sus parejas, compañeros(as), amigos(as), conocidos o los grupos a los que se pertenece o se desea pertenecer (Meningaud, et al., 2003). Por lo tanto, es muy probable que tales personas tengan identidades problemáticas, a saber, normativa y evasivo/difusa. Futuros estudios podrían evaluar la asociación entre trastornos de personalidad (ansiedad, depresión, trastorno obsesivo compulsivo), y los tipos de identidad en los pacientes de cirugía estética.

Para finalizar, otra hipótesis. Si la imagen corporal es fundamental en la identidad de un adolescente, y éstos basan generalmente su autoestima en su imagen corporal, la cual forma parte de su patrón de identidad, entonces aquella podría influir en la toma de muchas decisiones, entre 
ellas la elección de cirugía estética, sobre todo en los casos donde se tiene una autoimagen negativa y se prefieren más los tipos ideales de belleza corporal promovidos en un espacio social determinado.

\section{Manejo del terror y cirugía estética}

Junto a la imagen corporal, la dismorfia corporal, y los patrones de identidad, el manejo del terror es otra perspectiva psicosocial que también puede ayudar a predecir la elección de cirugía estética. La gestión del terror es una noción desarrollada para hacer referencia a la conciencia de la muerte y del envejecimiento, y al intento de luchar contra ello (Greenberg et al., 1986; Greenberg et al., 1997).

La teoría del manejo del terror (TMT) sostiene que la conciencia de la inevitabilidad de la muerte, o el miedo a la muerte, impulsa casi toda la actividad humana. Esta teoría también considera que los individuos que se ven a sí mismos como participantes activos y valiosos en espacios culturales significativos, les puede ayudar a amortiguar la ansiedad que resulta de reconocerse mortales (Greenberg et al., 1986).

La conciencia de ser mortales influye en muchos comportamientos humanos, y también en actitudes, opiniones, pensamientos, creencias y valores, por ejemplo, atracción interpersonal, juicios sobre transgresores morales, prejuicios, agresión, estimaciones del consenso social sobre las actitudes de uno, conformidad con los estándares y valores culturales, asunción de riesgos sin importar las consecuencias, etc. (Greenberg et al., 1997).

La conciencia sobre la mortalidad, y la importancia atribuida, abre una paradoja: tratar de evitarla/negarla, y darse cuenta que es inevitable. Es precisamente el deseo de querer vivir para siempre, y darse cuenta que no se puede, lo que aterra al ser humano y lo lleva a intentar escapar de este miedo eligiendo determinados mecanismos de defensa, por ejemplo, la fe religiosa y la autoestima, pero también se puede elegir la cirugía estética.

Muchos factores pueden ayudar a amortiguar el miedo a la muerte. Uno de ellos es la fe religiosa en la vida eterna después de la muerte. Otro es la autoestima individual que suele amortiguar la ansiedad ante la muerte (Greenberg, et al., 2008). Las creencias sobre la muerte también pueden amortiguar la ansiedad ante ésta, por ejemplo, quienes desde su propia visión cultural del mundo consideran que la muerte es algo natural, y que, por tanto, no se le debería tener miedo, podrían amortiguar más eficazmente tal miedo.

Sin embargo, la fe religiosa y la autoestima presentan un problema. Como visiones culturales del mundo, requieren la aprobación continua de otros para que funcione defensivamente de manera eficaz contra algo amenazante. En otras palabras, las instituciones u organizaciones que promueven la integración social deben tener el suficiente poder de integración sobre los sujetos que pretende integrar, de lo contrario, si existen dudas, por ejemplo, con respecto a la fe religiosa, el miedo a la muerte puede crecer (Birnbaum, et al., 2011).

Goldenberg et al., (2000), apoyado en la teoría de que una amplia gama de comportamientos humanos relacionados con el cuerpo proviene, en parte, de la ansiedad ante la muerte, sostienen que la preocupación por la muerte puede llevar a que los individuos actúen preservando la salud física de sus cuerpos, por ejemplo, alimentándose bien y haciendo ejercicio, pero también pueden estar obsesionados en cómo sus cuerpos se ven, huelen y funcionan en un espacio social, y en compararse con los estándares de belleza, mismos que buscarían alcanzar a través de la cirugía estética. Sobre esto último, Goldenberg y Arndt (2008) plantean la siguiente hipótesis: cuando los individuos son conscientes de la naturalidad de la muerte, y que no se debe tener miedo, tienden a realizar ejercicio y dietas saludables para vivir más tiempo; en cambio, cuando experimentan inconscientemente terror a la muerte, como defensa tienden a participar en actividades que simbolizan la inmortalidad, por ejemplo, tener descendencia y buscar el logro (Greenberg, et al., 1997), y podría ser también elegir la cirugía estética. 
Se dijo arriba que la autoestima también es un mecanismo para amortiguar el miedo a la muerte. Al respecto Harmon-Jones et al., (1997) plantean la siguiente hipótesis: quiénes ven a la muerte como algo natural e inevitable, tienen alta autoestima, y menor ansiedad ante la muerte, no elegirán la cirugía estética, elección que si tomarían quiénes tienen baja autoestima y alta ansiedad ante la muerte porque requieren alejarse de ella o demorarla simbólicamente.

Otra hipótesis de investigación interesante es la siguiente. Es posible que quiénes se someten a cirugía estética perciban un resultado positivo de auto imagen; con ello también pueden sentirse adaptados a los patrones culturales dominantes de la belleza física, lo que puede también aumentar su autoestima, y disminuir su ansiedad ante la muerte (Thorpe, et al., 2004). ¿Quiénes son los individuos que al someterse a cirugía estética mejoran su autoimagen, su autoestima y además bajan su nivel de ansiedad ante la muerte? Las investigaciones que arrojen luz sobre tal cuestión son necesarias no sólo para avanzar en el desarrollo del conocimiento sino para diseñar programas que coadyuven en el tratamiento psicosocial de estos individuos.

\section{Religión y cirugía estética}

Como se dijo arriba, una característica común de las religiones es que prometen una vida más allá de la muerte a sus seguidores. También se acepta que todo lo que Dios ha dado es algo bueno y no debe modificarse porque eso es pecado, por ejemplo, el cuerpo y la cara (Greeley y Hout, 2006). La creencia en el más allá suele funcionar como una especie de agencia sobrenatural que permite la trascendencia de la materialidad del cuerpo humano (Greenberg et al., 1986). Otro punto importante acerca de la religiosidad es que la seguridad ontológica que concede la fe religiosa también aumenta cuando estos individuos siguen disciplinadamente las reglas y normas que dictan las comunidades religiosas a que pertenecen. Así dicho, la seguridad en estas colectividades humanas viene por una doble vía: sentirse protegido por un ser sobrenatural, y sentirse aceptado y apoyado por los miembros de la comunidad religiosas de pertenencia (Behravan, 2018: 87).

Tomando en cuenta todo lo que hasta aquí se ha dicho, es posible plantear varias hipótesis. Una de ellas es la siguiente. Se ha dicho que la intención principal de la elección de cirugía estética es la búsqueda de la belleza, combatir el envejecimiento y mejorar la salud mental. Mirarse envejecer frente al espejo puede crear en los individuos la sensación de acercarse al final de la existencia, y motivarse a elegir la cirugía estética como manera de afrontar el problema. A diferencia de los individuos no religiosos, quienes creen en la vida después de la muerte y se sienten aceptados por los miembros de su congregación religiosa, su autoestima y autovaloración es alta, y ello les permite aceptar su cuerpo tal como es, y no desearían modificarlo mediante cirugía estética (Behravan, 2018: 90).

\section{Perfeccionismo y cirugía estética}

El perfeccionismo también es un trastorno de personalidad. En la última actualización del DSMV, American Psychiatric Association, el perfeccionismo ya forma parte del Trastorno obsesivo compulsivo (TOC) al definirlo como patrón general de preocupación por el orden, el perfeccionismo y el control mental e interpersonal. Un patrón duradero de experiencia interna y comportamiento que se desvía notablemente de las expectativas del individuo es el perfeccionismo, y se manifiesta en las áreas de cognición y afectividad. Por cognición se entiende las formas de percibirse e interpretarse a sí mismo, y a otras personas y eventos. La afectividad puede tenerse como el rango, intensidad, responsabilidad y adecuación de la respuesta emocional, el funcionamiento interpersonal y el control de los impulsos (Behravan, 2018: 99).

En general, los perfeccionistas se caracterizan por estar disgustados con su cuerpo (Ruggiero, et al., 2003) y con la sintomatología dismórfica (Hanstock y O'Mahony, 2002). Por lo anterior, los perfeccionistas tienden frecuentemente a transformar sus cuerpos, por ejemplo, mediante la alimentación (Cockell, et al., 2002), el culturismo (Davis y Scott-Robertson, 2000) y el ejercicio excesivo (Gulker, et al., 2001). De acuerdo con lo anterior, se puede suponer que los individuos 
con alto perfeccionismo también tienen entre sus consideraciones alterar su apariencia mediante la cirugía estética, sobre todo las partes que no se pueden modificar con dieta y ejercicio (nariz, labios, ojos, senos, etc.)

Para Sherry et al., (2003), la cirugía estética es una predisposición crónica de los perfeccionistas ante la insatisfacción corporal. Esta es una hipótesis interesante que se puede contrastar en realidades concretas. Además, para complejizar el problema, se pueden hacer intervenir otras variables, por ejemplo, las demográficas, y otras más que la creatividad de los investigadores permita.

\section{Conclusión}

Por razones de espacio, en este trabajo no se reflexiona sobre otras motivaciones psicosociales interesantes en relación con la cirugía estética, a saber, sentido de pertenencia, bienestar subjetivo, autoestima, conservadurismo, y toma de riesgos.

Cada uno de los supuestos aquí planteados en relación con las motivaciones psicosociales de cirugía estética pueden tenerse como una beta para futuras investigaciones en México y otras partes del mundo. Los investigadores, con su creatividad, también pueden plantear otros supuestos de investigación interesantes, y profundizar en el aspecto teórico.

En tanto forman parte de una investigación que se realiza actualmente, el presente trabajo no hace referencia a investigaciones realizadas en los últimos cuatro años, lo que también es una ventana de oportunidad para los investigadores interesados. Finalmente, las motivaciones psicosociales, por razones analíticas, pueden observarse por separado en relación con la cirugía estética; empero, en la realidad concreta suelen encontrarse imbricadas, influyéndose entre sí, lo que futuras investigaciones también podrían abordar. Por ejemplo, en la elección, o no, de agrandamiento de pechos, además de la imagen corporal pueden intervenir la autoestima y el trastorno dismórfico corporal, así como los valores religiosos, etc.

\section{Referencias bibliográficas}

American Psychiatric Association (2013). Diagnostic and Statistical Manual of Mental Disorders, 5th. Edition (DSM-5). American Psychiatric Association. Estados Unidos. https://doi.org/10.1176/appi.books.9780890425596.

Andreasen, Nancy C. y Bardach, Janusz (1977). Dysmorphophobia: symptom or disease? The American Journal of Psychiatry. Volumen 134, No. 6. Estados Unidos. (pp. 673-676) https://doi.org/https://doi.org/10.1176/ajp.134.6.673.

Askegaard, Søren; Cardel Gertsen, Martine y Langer, Roy. (2002). The Body Consumed: Reflexivity and Cosmetic Surgery. Psychology \& Marketing. Volumen 19, No. 10. Estados Unidos. (pp. 793-812). Extraído de https://scholar.google.com.mx/scholar?hl=es\&as sdt $=0 \% 2 \mathrm{C} 5 \&$ as vis $=1 \& \mathrm{q}=\mathrm{The}+\mathrm{Bod}$ $\mathrm{y}+$ Consumed $\% 3 \mathrm{~A}+$ Reflexivity + and + Cosmetic + Surgery\&btnG $=$

Behravan, Bita (2018). The Hidden Psycho-Social Dimensions of Cosmetic Surgery CrossCultural Studies of Cosmetic Surgery. Motivation among Iranian and German Women. Tesis de doctorado en filosofía. Universidad de Duisburg-Essen, Facultad de educación. Alemania. Extraído de https://duepublico2.unidue.de/servlets/MCRFileNodeServlet/duepublico derivate 00045903/DissBehravan.pd f.

Berzonsky, Michael D (1988). Self-theorist, identity status, and social cognition. En Lapsley, Daniel K. y Power, F. Clark. (Eds). Self, ego, and identity: integrative approaches. Springer-Verlag, Estados Unidos. https://doi.org/10.1007/978-1-4615-7834-5 12

Berzonsky, Michael D (1990). Self construction across the life-span: A process view of identity development. En Neimeyer, Greg J. y Neimeyer, Robert A. (Eds). Advances in personal 
construct psychology (vol 1, pp. 155-186). JAI Press. Estados Unidos. https://psycnet.apa.org/record/1992-98844-006.

Berzonsky, Michael D (1992). Identity Style and Coping Strategies. Journal of Personality. Volumen 60, No. 4. Estados Unidos (771-778). Extraído de https://www.researchgate.net/profile/Michael-

Berzonsky/publication/313190000 A process perspective on identity and stress ma nagement/links / 5e07b391a6fdcc283745e5e0/A-process-perspective-on-identity-andstress-management.pdf

Berzonsky, Michael D (2003). Identity style and well-being: Does commitment matter? Identity: An International Journal of Theory and Research. Volumen 3, No. 2. Inglaterra. (pp. 131-142) Extraído de https://www.researchgate.net/publication/240237125 Identity Style and WellBeing Does Commitment Matter.

Berzonsky, Michael D. y Ferrari, Joseph R (2009). A diffuse-avoidant identity processing style: Strategic avoidance or self-confusion? Identity: An International Journal of Theory and Research, Volumen 9, No. 2. Inglaterra. (pp. 145-158). Extraído de https://www.researchgate.net/publication/233448564 A Diffuse-

Avoidant Identity Processing Style Strategic Avoidance or Self-Confusion.

Berzonsky, Michael D. y Kuk, Linda S (2000). Identity Status, Identity Processing Style, and the Transition to University. Journal of Adolescent Research. Volumen 15, No. 1. (pp. 81 98). Estados Unidos. http://doi.org/10.1177/0743558400151005.

Berzonsky, Michael D. y Neimeyer, Greg J. (1994). Ego Identity Status and Identity Processing Orientation: The Moderating Role of Commitment. Journal of Research in Personality. Volumen 28, No. 4. Holanda. (pp. 425-435). https://doi.org/10.1006/irpe.1994.1030.

Birnbaum, Gurit; Hirschberger, Gilad y Goldenberg, Jamiel L (2011). Desire in the face of death: Terror management, attachment, and sexual motivation. Personal Relationships. Volumen 18, No. 1. Inglaterra. (pp. 1-19). https://doi.org/10.1111/j.14756811.2010.01298.x

Blum, Virginia L (2003). Flesh Wounds: culture of cosmetic surgery. University of California Press. Inglaterra. Extraído de https://www.anme.com.mx/libros/Flesh\%20Wounds\%20$\% 20 \mathrm{The} \% 20$ Culture $\% 20$ of $\% 20$ Cosmetic $\% 20$ Surgery $\% 20 \mathrm{II}$.pdf

Cash, Thomas F. (2004). Body image: past, present, and future. Body Image. Volumen 1, No. 1. Estados Unidos. (pp. 1-5). https://doi.org/10.1016/S1740-1445(03)00011-1

Cockell, Sarah J; Hewitt, Paul L; Seal, Brooke; Sherry, Simon; Goldner, Elliot M; Flett, Gordon L. y Remick, Ronald A (2002). Trait and Self-Presentational Dimensions of Perfectionism Among Women with Anorexia Nervosa. Cognitive Therapy and Research. Volumen 26, No. 6. Estados Unidos. (pp. 745-758). Extraído de https://www.researchgate.net/publication/328744825 Trait and selfpresentational dimensions of perfectionism among women with anorexia nervosa.

Crerand, Canice E; Phillips, Katharine A; Menard, William; y Fay, Christina. (2005). Nonpsychiatric medical treatment of body dysmorphic disorder. Psychosomatic. Volumen 46, No. 6. Holanda. (pp. 549-55). Extraído de https://www.ncbi.nlm.nih.gov/pmc/articles/PMC1351255/.

Davis, Caroline y Scott-Robertson, Lore (2000). A psychological comparison of females with anorexia nervosa and competitive male bodybuilders: body shape ideals in the extreme. Eating Behaviors. Volumen 1, No. 1. Holanda. (pp. 33-46). https://doi.org/10.1016/S1471-0153(00)00007-6.

Davis, Kathy. (2002). 'A Dubious Equality': Men, Women and Cosmetic Surgery. Body \& Society. Volumen 8, No. 1. Estados Unidos. (pp. 49-65). Extraído de 
http://citeseerx.ist.psu.edu/viewdoc/download?doi=10.1.1.992.5133\&rep=rep1\&type= pdf.

De Castro, Ana Lucia (2009). Cultura contemporánea, mass media y culto al cuerpo. La cuestión de las identidades. XXVII Congreso de la Asociación Latinoamericana de Sociología. Asociación Latinoamericana de Sociología. Argentina. Extraído de https://www.aacademica.org/000-062/2158

Feixa, Carles (2020). Identidad, Juventud y Crisis: el concepto de crisis en las teorías sobre la juventud. Revista Española de Sociología (RES). Volumen 29, No. 3. España. (pp. 11 26). https://doi.org/10.22325/fes/res.2020.72

Freud, Sigmund (1914-1918). Historia de una neurosis infantil (caso del "hombre de los lobos"). Librodot.com de acceso abierto en internet. Extraído de http://www.insumisos.com/M4T3R14L/BD/Freud-

Sigmund/Historia $\% 20 \mathrm{de} \% 20$ una $\% 20$ neurosis $\% 20$ infantil $\% 20$ (caso $\% 20 \mathrm{del} \% 20$ Hombre $\% 20 \mathrm{de} \% 20 \mathrm{los} \% 20$ Lobos).PDF

Goldenberg, Jamie L; y Arndt, Jamie. (2008). The implications of death for health: A terror management model of behavioral health promotion. Psychological Review, Volumen 115, No. 4. Estados Unidos. (pp. 1032-1053). http://doi.org/10.1037/a0013326.

Goldenberg, Jamie L; McCoy, Shannon K; Pyszczynski, Tom; Greenberg Jeff; y Solomon, Sheldon (2000). The body as a source of self-esteem: The effect of mortality salience on identification with one's body, interest in sex, and appearance monitoring. Journal of Personality and Social Psychology. Volumen 79, No.1. Estados Unidos. (pp. 118-130). https://doi.org/10.1037/0022-3514.79.1.118.

Greeley, Andrew y Hout, Michael (2006). The truth about conservative Christians. What they think and what they believe. University of Chicago Press. Estados Unidos.

Greenberg Jeff., Pyszczynski Tom. y Solomon, Sheldon (1986). The causes and consequences of a need for self-esteem: A terror management theory. En Baumeister, Roy F (Ed.). Public self and private self. Springer-Verlag. Estados Unidos. (pp. 189 -212). Extraído de https://doi.org/10.1007/978-1-4613-9564-5 10

Greenberg Jeff., Solomon Sheldon. y Arndt Jamie (2008). A basic but uniquely human motivation: Terror management. En Shah, James Y Gardner, Wendy L (Eds.). Handbook of motivation science. Guilford Press. Estados Unidos. Extraído de https://psycnet.apa.org/record/2008-00543-008

Greenberg, Jeff; Solomon, Sheldon, y Pyszczynski, Tom. (1997). Terror management theory of self-esteem and cultural worldviews: Empirical assessments and conceptual refinements. Advances in Experimental Social Psychology, Volumen 29. Estados Unidos. (pp. 61139). https://doi.org/10.1016/S0065-2601(08)60016-7.

Grogan, Sarah (1999). Body image: Understanding body dissatisfaction in men, women, and children. Routledge, Primera edición. Inglaterra. DOI: https://doi.org/10.4324/9780203004340

Gulker, M.G. Laskis, T.A. y Kuba, Sue A (2001). Do excessive exercisers have a higher rate of obsessive-compulsive symptomatology? Psychology, Health and Medicine. Volumen 6, No. 4. Inglaterra. (pp. 387-398). Extraído de https://doi.org/10.1080/13548500126535.

Hanstock, Tanya L. y O'Mahony, John F (2002). Perfectionism, acne and appearance concerns. Personality and Individual Differences. Volumen 32, No. 8. Inglaterra. (pp. 13171325). https://doi.org/10.1016/s0191-8869(01)00120-9.

Harmon-jones, Edie; Simon, Linda; Greenberg Jeff; Pyszczynski, Tom; Solomon, Sheldom y McGregor, Holly A (1997). Terror management theory and self-esteem: Evidence that increased self-esteem reduces mortailty salience effects. Journal of personality and social psychology, Volumen 72, No. 1. Estados Unidos. (pp. 24- 36). http://doi.org/10.1037//0022-3514.72.1.24 
ISAPS (2015). International Survey on Aesthetic/Cosmetic. Estados Unidos. Extraído de https://www.isaps.org/wp-content/uploads/2017/10/2016-ISAPS-Results-1.pdf

ISAPS (2018). International survey on aesthetic/cosmetic procedures. Estados Unidos. Extraído de https://www.isaps.org/wp-content/uploads/2020/10/ISAPS-GlobalSurvey-Results-2018-1.pdf

ISAPS (2019). International survey on aesthetic/cosmetic procedures. Estados Unidos. Extraído de https://www.isaps.org/wp-content/uploads/2020/12/Global-Survey2019.pdf.

Marcia, James E (1966). Development and validation of ego identity status. Journal of Personality and Social Psychology. Volumen 3, No. 5. Estados Unidos. (pp. 551-558). Extraído de https://www.researchgate.net/profile/JamesMarcia/publication/17245812 Development and Validation of Ego Identity Status/1 inks/0046351912fd706fd4000000/Development-and-Validation-of-Ego-IdentityStatus.pdf.

Mead, George Herbert. (1973 [1932]). Espíritu, persona y sociedad. Desde el punto de vista del conductismo social. Paidos. Buenos Aires. Extraído de https://www.academia.edu/11620336/1Mead_Espi ritu persona_y sociedad 23166 1

Meningaud, Jean Paul; Benadiba Laurent; Servant, Jean Marie; y Herve Christian (2001). Depression, anxiety and quality of life among scheduled cosmetic surgery patients: multi center prospective study. Journal of maxillofacial surgery. Volumen 29, No. 3. Austria. (pp. 177-180). https://doi.org/10.1054/jcms.2001.0213

Meningaud, Jean Paul; Benadiba, Laurent; Servant, Jean Marie; Hervé, Christian; Bertrand, Jacques Charles; y Pelicier, Yves (2003). Depression, anxiety and quality of life: Outcome 9 months after facial cosmetic surgery. Journal of Cranio-Maxillofacial Surgery. Volumen 31, No. 1. Wien, Austria. (pp. 46-50). https://doi.org/10.1016/S10105182(02)00159-2

Mercado Maldonado, Asael y Hernández Oliva, Alejandrina V (may-ago 2010). El proceso de construcción de la identidad colectiva. Convergencia, revista de ciencias sociales. Volumen 17, No. 53. México. (pp. 229-251). Extraído de http://www.scielo.org.mx/scielo.php?script=sci arttext\&pid=S140514352010000200010.

Nurmi Jari-Erik; Berzonsky, Michael D; Tammi, Kari; y Kinney Andrew (1997). Identity processing orientation, cognitive and behavioral strategies, and well-being. International Journal of Behavioral Development. Volume 21, No. 3. Estados Unidos. (pp. 555-570). https://doi.org/10.1080/016502597384785.

Phillips, Katharine A (1993). Body dysmorphic disorder: 30 cases of imagined ugliness. American Journal Psychiatry. Volumen 150, No. 2. Estados Unidos. (pp. 302-308). https://doi.org/10.1176/ajp.150.2.302

Phillips, Katharine A; Grant, Jon; Siniscalchi, Jason; y Albertini, Ralph S (2001). Surgical nonpsychiatric medical treatment of patients with body dysmorphic disorder. Psychosomatics. Volumen 42, No. 6. Holanda. (pp. 504-510). https://doi.org/10.1176/appi.psy.42.6.504

Phillips, Katharine A y Diaz, S. F (1997). Gender differences in body dysmorphic disorder. The Journal of Nervous and Mental Disease. Volumen 185, No. 9. Estados Unidos (pp. 570-577). https://doi.org/10.1097/00005053-199709000-00006

Portal Ariosa, Ana María (1991). Diversas perspectivas en la construcción teórica de la identidad: una bibliografía básica. Alteridades. Volumen 1, No. 2. México. (pp. 122-125). Extraído de https://www.redalyc.org/pdf/747/74745539015.pdf 
Ruggiero, Giovani Maria; Levi, Debora; Ciuna, Arcanjelo; y Sassaroli, Sandra (2003). Stress situation reveals an association between perfectionism and drive for thinness. International Journal of Eating Disorders. Volumen 34, No. 2. Estados Unidos. (pp. 220-226). https://doi.org/10.1002/eat.10191.

Sarwer, David B; Cash, Thomas F., Magee, Learne; Williams, Emily Fleming; Thompson, J. Kevin; Roehrig, Megan; Tantleff-Dunn, Stacey., Agliata, Alison Kanter; Wilfley, Denise E; Amidon, Amy D; et al., (2005). Female college students and cosmetic surgery: an investigation of experiences, attitudes, and body image. Plastic and Reconstructive Surgery. Volumen 115, No. 3. Estados Unidos. (pp. 931-938). https://doi.org/10.1097/01.prs.0000153204.37065.d3.

Sarwer, David B; Pertschuk, Michael J; Wadden, Thomas A; y Whitaker Linton A (1998). Psychological investigations in cosmetic surgery: a look back and a look ahead. Plastic and Reconstructive Surgery. Volumen 101, No. 4. Estados Unidos. (pp.1136-1142). Extraído de

https://journals.lww.com/plasreconsurg/Abstract/1998/04000/Psychological Investiga tions in Cosmetic Surgery .40.aspx

Sherry, Simon B; Hewitt, Paul L; Lee-Baggley, Dayna L; Flett, Gordon L; y Besser, Avi (2004). Perfectionism and thoughts about having cosmetic surgery performed. Journal of Applied Biobehavioral Research. Volumen 9, No. 4. Estados Unidos (pp. 244-257). https://doi.org/10.1111/j.1751-9861.2004.tb00103.x.

Thoits, Peggi A (1995). Stress, coping, and social support processes: Where are we? What next? Journal of Health and Social Behavior. Volumen 35, No. 35 (edición especial: Forty Years of Medical Sociology: The State of the Art and Directions for the Future). Estados Unidos. (pp. 53-79). Extraído de https://www.asanet.org/sites/default/files/savvy/images/members/docs/pdf/special/j hsb/jhsb extra 1995 Article 3 Thoits.pdf.

Thorpe, Susan J; Ahmed, Beena; y Steer, Kent (2004). Reasons for undergoing cosmetic surgery: A retrospective study. Sexualities, Evolution y Gender. Volumen 6, No. 2. Inglaterra. (pp. 75-96). http://doi.org/10.1080/14616660412331303857.

Veale, David; Boocock, Ann; Gournay, Kevin; Dryden, Windy; Shah, Fozia; Willson, Robert; y Jessica Walburn, Jessica (1996). Body dysmorphic disorder: a survey of fifty cases. British Journal of Psychiatry. Volumen 169, No. 2. Inglaterra. (pp. 196-201). http://doi.org/10.1192/bip.169.2.196.

Falakolaflaki, Sima y Hormozi, Atousa. (2015). A Comparative Study of Identity Style in Deaf and Normal Adolescents. Mediterranean Journal of Social Sciences. Volumen 6, No. 2. Inglaterra. (pp. 662-667). http://doi.org/10.5901/mjss.2015.v6n2s1p662 